\title{
Adjunctive corticosteroids for the treatment of Pneumocystis jiroveci pneumonia in patients with HIV: A meta-analysis
}

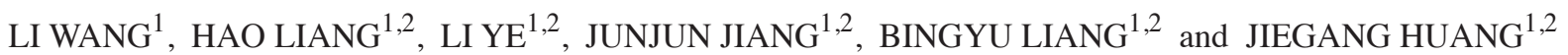 \\ ${ }^{1}$ Guangxi Key Laboratory of AIDS Prevention and Treatment; ${ }^{2}$ School of Public Health, \\ Guangxi Medical University, Nanning, Guangxi 530000, P.R. China
}

Received October 26, 2014; Accepted November 5, 2015

DOI: $10.3892 / \mathrm{etm} .2015 .2929$

\begin{abstract}
The present study aimed to evaluate the effects of adjunctive corticosteroid treatment on Pneumocystis jiroveci pneumonia in patients with human immunodeficiency virus (HIV). A literature search of relevant randomized controlled trials (RCTs) published prior to March 2014 was performed using a number of websites, including PubMed, EMbase and Ovid, using the following keywords: Corticosteroids, glucocorticoide, cortisol, corticosterone, HIV/acquired immunodeficiency syndrome, $P$. jiroveci pneumonia, and PCP. All RCTs investigating the use of adjunctive corticosteroids for the treatment of $P$. jiroveci pneumonia in patients with HIV were evaluated in the present study. Stata 11.0 software was used to calculate the relative risk (RR) and $95 \%$ confidence interval (CI) following tests for consistency and potential biases. Six RCTs investigating a total of 548 patients were evaluated in the present meta-analysis. The experimental groups $(n=270)$ demonstrated a mortality rate of $15.2 \%(n=41)$; as compared with $27.7 \%(n=77)$ in the control groups $(n=278)$. The present meta-analysis demonstrated that the RR and 95\% CI were 0.55 and $0.35-0.85(\mathrm{P}<0.05)$, respectively, following treatment with adjunctive corticosteroids. This result indicated that patients in the experimental group had a 0.55 times reduced risk of mortality compared with the control group. Therefore, the results of the present meta-analysis demonstrated that the administration of adjunctive corticosteroids for the treatment of $P$. jiroveci pneumonia in patients with HIV may reduce the mortality rate of patients in the early phase of the disease.
\end{abstract}

Correspondence to: Professor Jiegang Huang, School of Public Health, Guangxi Medical University, 22 Shuangyong Road, Nanning, Guangxi 530000, P.R. China

E-mail: katooo@163.com

Key words: human immunodeficiency virus, meta-analysis, adjunctive corticosteroids, Pneumocystis jiroveci pneumonia

\section{Introduction}

Acquired immunodeficiency syndrome (AIDS) is among the most complex diseases in the field of medicine. The incidence of Pneumocystis jiroveci pneumonia (PCP) has increased significantly and has become the most common opportunistic infection among patients with human immunodeficiency virus (HIV) (1). PCP is the most common cause of AIDS-related mortality, and the mortality rate of patients with AIDS that contract PCP in the early stages of the disease increases to $10-20 \%$ as the necessity for mechanical ventilation is significantly increased (2). Based on the results of five randomized controlled trials (RCTs), the use of corticosteroids was recommended for the treatment of patients co-infected with HIV and PCP by an expert panel in 1990 (3). Adjunctive corticosteroid treatment refers to the administration of corticosteroids in combination with sulfamethoxazole-trimethoprim (SMZ-TMP) or pentamidine $(3,4)$. Corticosteroids may be categorized as long-acting, middle-acting or short-acting agents. The primary therapeutic agents used to treat PCP in patients with HIV include prednisone and prednisolone (5). On the basis of the results of previous clinical trials, a systematic review was conducted by Briel et al (5) in 2006, which demonstrated the feasibility of adjunctive corticosteroid treatment for the treatment PCP in patients co-infected with HIV. Adjunctive corticosteroid therapy was considered as an alternative to SMZ-TMP alone, and effectively improved survival in moderate to severe cases, reducing complications such as pneumothorax and respiratory failure (6). Therefore, adjunctive corticosteroid therapy has been recommended by the American CDC Guidelines to treat PCP associated with HIV-1 infection (6). However, corticosteroid therapy may increase the occurrence of opportunistic infections, by causing deterioration of cell-mediated immunity $(7,8)$. Thus, the present meta-analysis aimed to evaluate the effects of adjunctive corticosteroid treatment for PCP in patients co-infected with HIV and to provide suggestions for clinical practice.

\section{Materials and methods}

Search methods. In order to analyze relevant RCTs, a search of the literature from the earliest available date to March 2014 


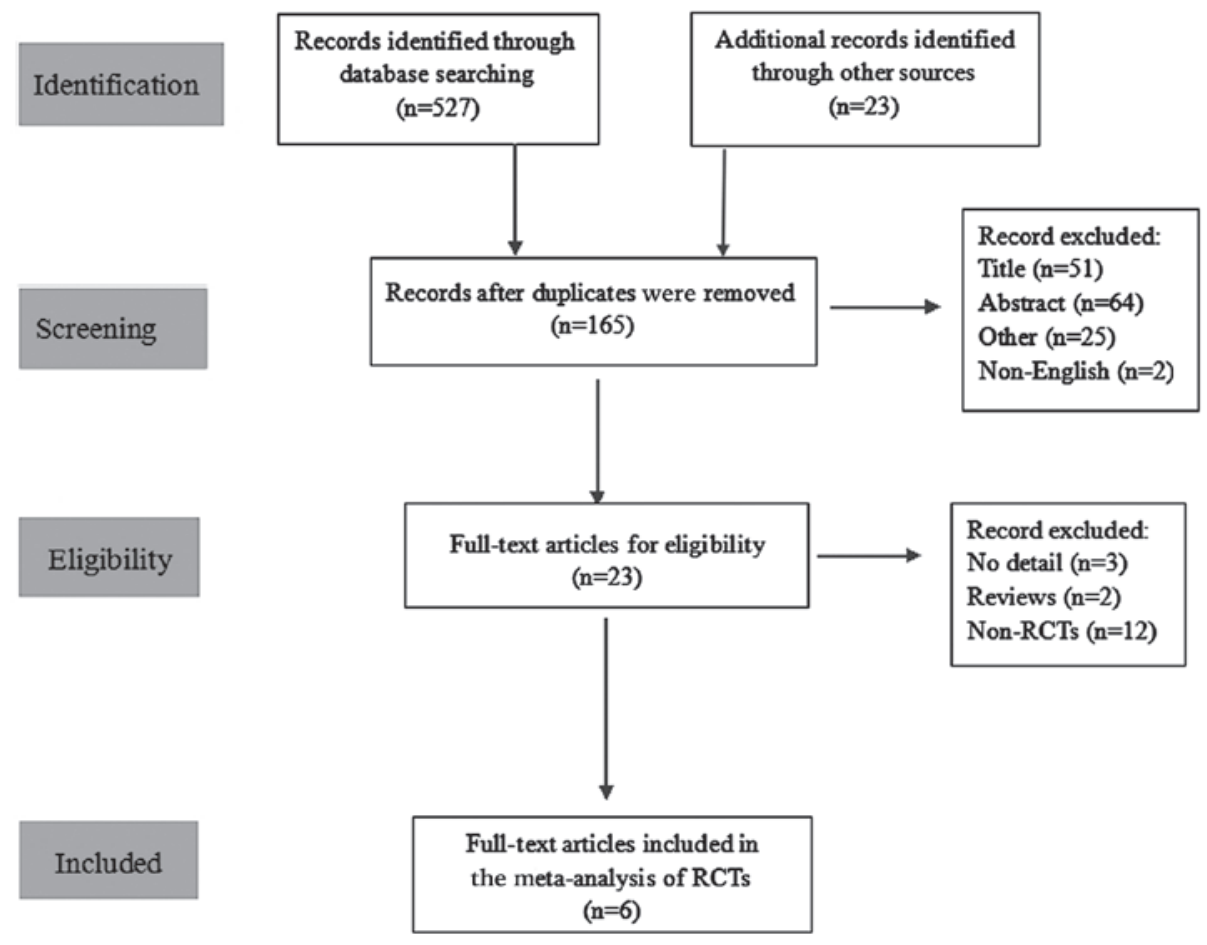

Figure 1. Flow chart summarizing the literature search.

was performed using various websites, including PubMed (http://www.ncbi.nlm.nih.gov/pmc/), Embase (https://www. elsevier.com/solutions/embase-biomedical-research) and Ovid (http://gateway.ovid.com/), using the following keywords: Corticosteroids; glucocorticoide; cortisol; corticosterone; HIV/AIDS; P. jiroveci pneumonia; and PCP.

Inclusion criteria. The following inclusion criteria were used in the present meta-analysis: i) Only RCT; ii) object of study, PCP in patients with HIV; iii) intervening measure, adjunctive corticosteroids treatment for patients with HIV in addition to the standardized treatment in the experimental group, and the use of placebo based on the standardized treatment, or standardized treatment alone administered to the control group; iv) patient reported outcomes, the mortality rate of patients following 1 month (28-35 days); and v) language, English.

Exclusion criteria. Studies that met the inclusion criteria were excluded according to the following criteria: Experimental design was not strict (i.e. lacked a control group); sample data was unclear or missing and experimental data could not be extracted; the information was not accurate and the data could not be used; or the content of the literature was repetitive.

Assessment of study quality. Standard Jadad scoring was used to assess the methodological quality of the included RCTs, based on the adequacy of randomization, blinding and follow-up, with a maximum score of 5 points. A score of 0-2 indicated low quality, whereas a score of 3-4 indicated higher quality and a score of 5 denoted high quality.

Data synthesis and meta-analysis. In order to calculate the relative risk (RR) with $95 \%$ confidence interval (CI), data were analyzed using Stata 11.0 software (StataCorp LP, College Station, TX, USA). The $\chi^{2}$ test was used to analyze the heterogeneity of the RCTs. P $>0.05$ indicated that there was no statistically significant heterogeneity, therefore a fixed-effects model was applied using the Mantel-Haenzel (M-H) method; whereas $\mathrm{P}<0.05$ indicated statistically significant heterogeneity, therefore a random-effects model was applied using the method proposed by DerSimonian and Laird (9).

Evaluating bias. In the present meta-analysis, potential publication bias was examined using a funnel plot. The symmetrical characteristics of the funnel plot were evaluated using the test proposed by Begg and Mazumdar (10) where a symmetrical result demonstrated no publication bias, and asymmetry indicated potential publication bias.

\section{Results}

Characteristics of included RCTs. A total of 550 studies were initially included; however, 527 were subsequently excluded due to duplicate content $(n=385)$, an irrelevant study topic, abstract or other dependency $(n=140)$ or because they were not published in English (n=2) (Fig. 1). Following detailed examination of the remaining 23 studies and the exclusion of citations that lacked sufficient detail or were not RCTs, six RCTs that met the inclusion criteria (11-16) were finally identified and the meta-analysis was performed. Table I outlines the RCTs included in the present meta-analysis.

Quality assessment of the included trials. The six included studies were all RCTs, four of which were double-blinded $(11,12,15,16)$ and two were not $(13,14)$. The respective control groups received placebo $(11,12,15,16)$ or null $(13,14)$ treatment 
Table II. Quality assessment of the included trials.

\begin{tabular}{lllc}
\hline RCT (ref.) & Randomization & Blinding & Jadad \\
\hline Terblanche et al $(11)$ & Adequate & Double-blind & 5 \\
Gagnon et al $(12)$ & Unclear & Unclear & 3 \\
Bozzette et al $(13)$ & Adequate & Non-blind & 3 \\
Nielsen et al $(14)$ & Adequate & Non-blind & 3 \\
Montaner et al $(15)$ & Adequate & Unclear & 4 \\
Walmsley et al $(16)$ & Adequate & Unclear & 4
\end{tabular}

No patient dropout or withdrawal occurred in any of the studies. RCT, randomized controlled trial.

(Table II). No significant differences were determined between the characteristics from the six trials. No patient withdrawal or dropout occurred in the six included trials. Jadad scores were as follows: $3(n=3), 4(n=2)$ and $5(n=1)$.

Meta-analysis. Six RCTs were enrolled in the present meta-analysis, which included a total of 548 patients with HIV suffering from PCP. The duration of observation was $\sim 1$ month (28-37 days), defined in terms of the patients' mortality rate. A total of were enrolled in The experimental groups, which contained 270 patients, demonstrated a mortality rate of $15.2 \%$ $(n=41)$, as compared with $27.7 \%(n=77)$ in the control groups, $(\mathrm{n}=278)$. A heterogeneity test demonstrated that $\chi^{2}=6.97$ $\left(\mathrm{P}=0.223 ; \mathrm{I}^{2}=28.3 \%\right)$, therefore no significant heterogeneity was detected and the M-H method was employed. Subsequent meta-analysis demonstrated that the experimental group had a significantly decreased risk of mortality (0.55 times; $\mathrm{P}<0.05$ ), as compared with the control group ( RR, 0.55; 95\% CI, 0.35-0.85) (Fig. 2).

Publication bias. Funnel plot analysis of publication bias in the six RCTs demonstrated that all six trials were present in the funnel plot and the majority were in the middle or top sections (Fig. 3). Funnel chart linear regression showed the bias coefficient was $\mathrm{t}=0.38[\mathrm{Pr}>|\mathrm{t}|=0.707$ (continuity corrected)], therefore the result was not statistically significant. The $95 \%$ CI was -3.52 to 2.34. The funnel plot was considered symmetrical, indicating no publication bias.

Sensitivity analysis. Sensitivity analysis of the included RCTs was performed by altering the inclusion criteria, excluding low quality RCTs and employing different statistical methods for analysis, using Stata 11.0 software. The results demonstrated that there were minor differences between the studies and the exclusion of any one study had minimal effects on the results (Fig. 4). When the random-effects model was used, the RR was 0.55 (95\% CI, 0.35-0.85), approximated to the fixed-effects model, indicating a small but statistically insignificant heterogeneity among the included studies.

\section{Discussion}

The effective treatment of AIDS and its complications remains a complex worldwide problem. The incidence of PCP has 


\begin{tabular}{|c|c|c|}
\hline Study ID & $\mathrm{RR}(95 \% \mathrm{Cl})$ & Weight $\%$ \\
\hline Terblanche AJ (2008) & $0.81(0.51,1.29)$ & 36.70 \\
\hline Gagnon S (1990) & $0.31(0.11,0.85)$ & 14.18 \\
\hline Sattler FR (1990) & $0.48(0.26,0.89)$ & 28.12 \\
\hline Nielsen TL (1992) & $0.21(0.05,0.91)$ & 7.97 \\
\hline Montaner JS (1990) & $3.16(0.14,72.84)$ & 1.88 \\
\hline Walmsley S (1995) & $0.63(0.19,2.07)$ & 11.15 \\
\hline Overall $\left(I_{2}=28.3 \%, p=0.223\right)$ & $0.55(0.35,0.85)$ & 100.00 \\
\hline
\end{tabular}

Figure 2. Mantel-Haenzel model for meta-analysis.RR, relative ratio; CI, confidence interval.

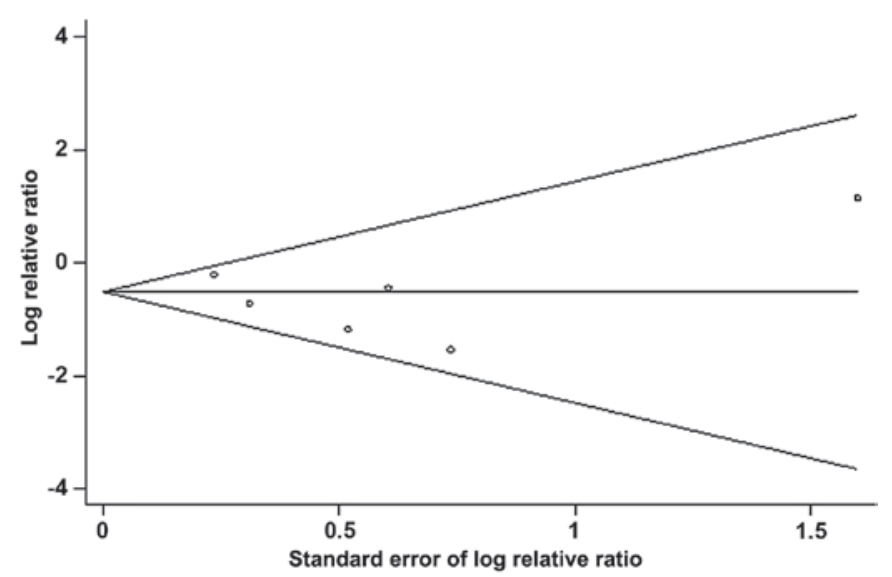

Figure 3. Begg's funnel plot analysis of the six included trials to investigate potential publication bias, with $95 \%$ confidence limits.

increased significantly and it has become the most common opportunistic infection in patients with HIV $(17,18)$. Adjunctive corticosteroids were initially suggested as a treatment for PCP in patients with HIV in 1990, and current treatment guidelines remain in agreement with this consensus statement (19).

Six RCTs with 548 cases of PCP in patients with HIV were enrolled in the present meta-analysis. The smallest sample size was 23 patients and the largest was 253 patients. The intervening measure was the use of adjunctive corticosteroids for the treatment of PCP in patients with HIV, with SMZ-TMP or pentamidline used as the standard treatment. Various types of control were used: Bozzette et al (13) and Nielsen et al (14) used blank controls, whereas the remaining RCTs $(11,12,15,16)$ used placebo treatments. The results of one study differed from the other five RCTs. In the study conducted by Montaner et al (15) the RR was 3.16, suggesting that patients in the experimental group had a risk of mortality that was 3.16 times higher compared with the control group. Notably, the remaining 5 RCTs all indicated that the use of adjunctive corticosteroids for the treatment of PCP in patients with HIV may reduce patient mortality. The results of the

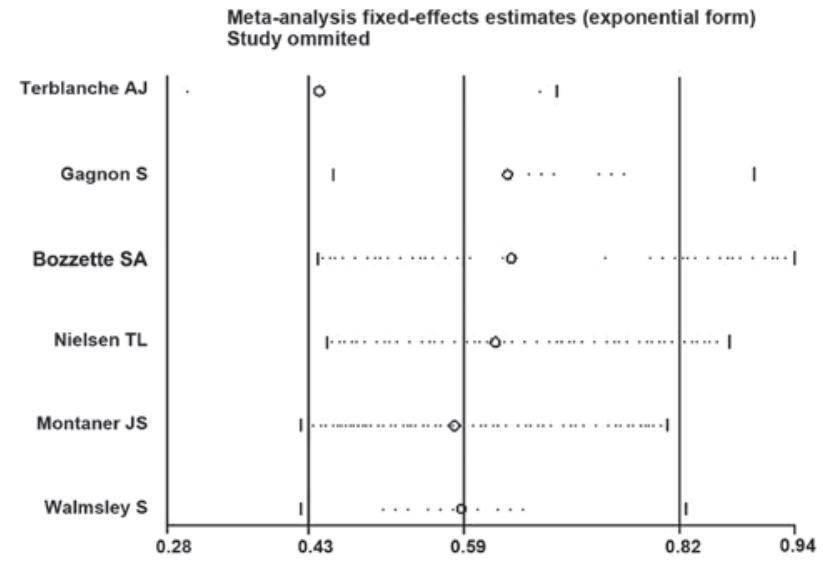

Figure 4. Sensitivity analysis of the included studies.

present meta-analysis demonstrated that the risk of mortality in the experimental group was 0.55 times lower compared with the control group, indicating that the use of adjunctive corticosteroids for the treatment of PCP in patients with HIV may reduce mortality in the early phase of the disease.

As bias may have an effect on the credibility of the present meta-analysis, potential biases were analyzed. Publication bias is the most common bias to consider when conducting meta-analyses as positive results are more frequently published. In the present meta-analysis, potential publication bias was examined using a funnel plot. The funnel plot analysis of the 6 RCTs indicated that all the trials were represented in the plot and the majority were in the middle or top sections. Furthermore, the results of the linear regression indicated that the funnel plot was symmetrical, therefore no publications bias was detected in the present meta-analysis. Two of the studies $(15,16)$ enrolled in the present meta-analysis used SMZ-TMP or pentamidline as standard treatment, whereas the others (11-14) used SMZ-TMP. The results of the heterogeneity test demonstrated that there was little heterogeneity between them $\left(\mathrm{P}=0.223 ; \mathrm{I}^{2}=28.3 \%\right)$. Furthermore, the results of the sensitivity analyses demonstrated that this did not have any 
influence on the results of the meta-analysis even following the exclusion of any one study. These results demonstrated that the difference between using SMZ-TMP/pentamidline and SMZ-TMP monotherapy was within the acceptable limits, therefore the results of the six RCTs could be combined for analysis. The results did not change when a random-effects model was used, demonstrating that there was minimal publication bias, and that any potential bias would have no substantial influence on the results of the present meta-analysis.

The results of the present meta-analysis indicated that the use of adjunctive corticosteroids for the treatment of PCP in patients with HIV may reduce mortality in the early stages of the disease. Therefore, adjunctive corticosteroids may be considered for the treatment of PCP in patients with HIV. However, there were a number of limitations to the present meta-analysis: An insufficient number of RCTs were analyzed; the sample sizes of certain studies were too low; and there were confounding factors, such as the origin of literature and the quantity of information, as not all six of the RCTs were double-blinded studies. Therefore, the conclusions of the present meta-analysis have certain limitations. Future studies will require RCTs with larger sample sizes in order to ensure the analyses of the effects of adjunctive corticosteroids on $\mathrm{PCP}$ in patients with HIV are more comprehensive, leading to a more reliable conclusion.

\section{Acknowledgements}

The present study was supported by the National Natural Science Foundation of China (grant no. 81360259), the Extracurricular Scientific Research Project of Guangxi Medical University (grant no. WLXSZX1411; Guangxi, China) and the Guangxi University Science and Technology Research Project (grant no. KY2015ZD026).

\section{References}

1. Kaplan JE, Hanson D, Dworkin MS, Frederick T, Bertolli J, Lindegren ML, Holmberg S and Jones JL: Epidemiology of human immunodeficiency virus-associated opportunistic infections in the United States in the era of highly active antiretroviral therapy. Clin Infect Dis 30 (Suppl 1): S5-S14, 2000.

2. Curtis JR, Yarnold PR, Schwartz DN, Weinstein RA and Bennett CL: Improvements in outcomes of acute respiratory failure for patients with human immunodeficiency virus-related Pneumocystis carinii pneumonia. Am J Respir Crit Care Med 162: 393-398, 2000

3. The National Institutes of Health-University of California Expert Panel for Corticosteroids as Adjunctive Therapy for Pneumocystis Pneumonia: Consensus statement on the use of corticosteroids as adjunctive therapy for pneumocystis pneumonia in the acquired immunodeficiency syndrome. N Engl J Med 323: 1500-1504, 1990.

4. Klein NC, Duncanson FP, Lenox TH, Forszpaniak C, Sherer CB, Quentzel H, Nunez M, Suarez M, Kawwaff O, Pitta-Alvarez A, et al: Trimethoprim-sulfamethoxazole versus pentamidine for Pneumocystis carinii pneumonia in AIDS patients: Results of a large prospective randomized treatment trial. AIDS 6: 301-305, 1992.
5. Briel M, Bucher HC, Boscacci R and Furrer H: Adjunctive corticosteroids for Pneumocystis jiroveci pneumonia in patients with HIV-infection. Cochrane Database Syst Rev 3: CD006150, 2006.

6. Panel on Opportunistic Infections in HIV-Infected Adults and Adolescents: Guidelines for Prevention and Treatment of Opportunistic Infections in HIV-Infected Adults and Adolescents: Recommendations from the Centers for Disease Control and Prevention, the National Institutes of Health, and the HIV Medicine Association of the Infectious Diseases Society of America. http://aidsinfo.nih.gov/contentfiles/lvguidelines/adult_ oi.pdf. Accessed November 20, 2015.

7. Wolfe F, Caplan L and Michaud K: Treatment for rheumatoid arthritis and the risk of hospitalization for pneumonia: Associations with prednisone, disease-modifying antirheumatic drugs, and anti-tumor necrosis factor therapy. Arthritis Rheum 54: 628-634, 2006.

8. Ko JH, Peck KR, Lee WJ, Lee JY, Cho SY, Ha YE, Kang CI, Chung DR, Kim YH, Lee NY, et al: Clinical presentation and risk factors for cytomegalovirus colitis in immunocompetent adult patients. Clin Infect Dis 60: e20-e26, 2015.

9. DerSimonian R and Laird N: Meta-analysis in clinical trials. Control Clin Trials 7: 177-187, 1986

10. Begg CB and Mazumdar M: Operating characteristics of a rank correlation test for publication bias. Biometrics 50: 1088-1101, 1994.

11. Terblanche AJ,GreenRJ,RheederPandWittenberg DF: Adjunctive corticosteroid treatment of clinical Pneumocystis jiroveci pneumonia in infants less than 18 months of age - a randomised controlled trial. S Afr Med J 98: 287-290, 2008.

12. Gagnon S, Boota AM, Fischl MA, Baier H, Kirksey OW and La Voie L: Corticosteroids as adjunctive therapy for severe Pneumocystis carinii pneumonia in the acquired immunodeficiency syndrome. A double-blind, placebo-controlled trial. N Engl J Med 323: 1444-1450, 1990.

13. Bozzette SA, Sattler FR, Chiu J, Wu AW, Gluckstein D, Kemper C, Bartok A, Niosi J, Abramson I, Coffman J, et al; California collaborative treatment group: A controlled trial of early adjunctive treatment with corticosteroids for Pneumocystis carinii pneumonia in the acquired immunodeficiency syndrome. N Engl J Med 323: 1451-1457, 1990.

14. Nielsen TL, Eeftinck Schattenkerk JK, Jensen BN, Lundgren JD, Gerstoft J, van Steenwijk RP, Bentsen K, Frissen PH, Gaub J, Orholm M, et al: Adjunctive corticosteroid therapy for Pneumocystis carinii pneumonia in AIDS: A randomized European multicenter open label study. J Acquir Immune Defic Syndr 5: 726-731, 1992.

15. Montaner JS, Lawson LM, Levitt N, Belzberg A, Schechter MT and Ruedy J: Corticosteroids prevent early deterioration in patients with moderately severe Pneumocystis carinii pneumonia and the acquired immunodeficiency syndrome (AIDS). Ann Intern Med 113: 14-20, 1990.

16. Walmsley S, Levinton C, Brunton J, Muradali D, Rappaport D, Bast M, Spence D and Salit I: A multicenter randomized double-blind placebo-controlled trial of adjunctive corticosteroids in the treatment of Pneumocystis carinii pneumonia complicating the acquired immune deficiency syndrome. J Acquir Immune Defic Syndr Hum Retrovirol 8: 348-357, 1995.

17. Thomas $\mathrm{CF} \mathrm{Jr}$ and Limper AH: Pneumocystis pneumonia. N Engl J Med 350: 2487-2498, 2004.

18. Ewald H, Raatz H, Boscacci R, Furrer H, Bucher HC and Briel M: Adjunctive corticosteroids for Pneumocystis jiroveci pneumonia in patients with HIV infection. Cochrane Database Syst Rev 4: D6150, 2015.

19. Benson CA, Kaplan JE, Masur H, Pau A and Holmes KK; CDC; National Institutes of Health; Infectious Diseases Society of America: Treating opportunistic infections among HIV-infected adults and adolescents: Recommendations from CDC, the National Institutes of Health and the HIV Medicine Association/Infectious Diseases Society of America. MMWR Recomm Rep 53 (RR-15): 1-112, 2004. 